Ramirez-Asis, E., Espinoza Maguiña, M., Esquivel Infantes, S. \& Naranjo-Toro, M. (2020). Inteligencia emocional, competencias y desempeño del docente universitario: Aplicando la técnica mínimos cuadrados parciales SEM-PLS. Revista Electrónica Interuniversitaria de Formación del Profesorado, 23(3), 99-114.

\title{
Inteligencia emocional, competencias y desempeño del docente universitario: Aplicando la técnica mínimos cuadrados parciales SEM-PLS.
}

\author{
Edwin Ramirez-Asis ${ }^{(1)}$, Marcos Espinoza Maguiña ${ }^{(1)}$, Santos Esquivel Infantes ${ }^{(2)}$, Miguel \\ Naranjo-Toro ${ }^{(3)}$ \\ (1) Universidad Nacional Santiago Antúnez de Mayolo, Perú. ${ }^{(2)}$ Universidad San Pedro, Perú. \\ (3) Universidad Técnica del Norte, Ecuador.
}

\section{Resumen}

La relevancia de las emociones en la enseñanza es ahora un aspecto ampliamente estudiado, aunque se está deliberando sobre la forma en que los docentes pueden identificar, regular y controlar sus emociones, el estudio propone cómo se puede incorporar la inteligencia emocional en la enseñanza mediante las competencias para lograr un mejor desempeño docente. Se utilizó la técnica de la encuesta recopilando datos de 244 docentes universitarios a través de un cuestionario mediante el muestreo aleatorio simple. Los datos se han analizado mediante el modelado de ruta utilizando el enfoque de mínimos cuadrados parciales (PLS) del modelo de ecuaciones estructurales SEM con el software SmartPLS 3.3.0, los hallazgos revelaron que el modelo tiene un impacto significativo en el desempeño docente, los coeficientes de determinación fueron; habilidades del docente $\left(r^{2}=0.703\right)$ actitud del docente $\left(r^{2}=0.661\right)$, conocimientos del docente $\left(r^{2}=0.648\right)$ y para el desempeño del docente $\left(r^{2}=0.733\right)$. con un error cuadrático medio de aproximación (SRMR) de 0.056 que hace relevante el modelo confirmatorio. Por lo tanto, se demostró estadísticamente que la inteligencia emocional tiene un fuerte impacto en las competencias de los docentes, lo que a su vez contribuye en el desempeño del docente universitario.

\section{Palabras clave}

Habilidades; madurez emocional; sensibilidad emocional; smartPLS. 


\title{
Emotional intelligence, competencies and performance of the university professor: using the SEM-PLS partial least squares technique.
}

\begin{abstract}
The relevance of emotions in teaching is now a widely studied aspect, although the way in which teachers can carry out, regulate and control their emotions is being discussed, the study proposes how emotional intelligence can be incorporated into teaching through the competences to achieve better teaching performance. The survey technique was used, collecting data from 244 university teachers through a questionnaire using simple random sampling. The data has been analyzed using partial least squares path modeling (PLS), approach of the SEM structural equation model with the SmartPLS 3.3.0 software, the findings revealed that the model has a significant impact on teaching performance, the determination coefficients were; teacher skills $\left(r^{2}=0.703\right)$ teacher attitude $\left(r^{2}=0.661\right)$, teacher knowledge $\left(r^{2}=0.648\right)$ and for teacher performance $\left(r^{2}=0.733\right)$. with a root mean square error of approximation (SRMR) of 0.056 that makes the confirmatory model relevant. Therefore, it was statistically demonstrated that emotional intelligence has a strong impact on teachers' competences, which in turn contributes to the professor performance.
\end{abstract}

\section{Key words}

Skills; emotional maturity; emotional sensitivity; smartPLS.

\section{Introducción}

En la actualidad son requeridos profesionales con habilidades sociales desarrolladas, capacidad de ajustarse a diferentes contextos para lograr un buen trabajo en equipo, la inteligencia emocional se manifiesta y fortalece a los equipos (Ovans, 2015; Ansari y Malik, 2017). Asimismo, los cambios normativos en la educación superior están generando una transición de efectivo a enseñanza afectivo (Uitto, Jokikokko y Estola, 2015). Adicionalmente el docente universitario obtiene satisfacción personal y gratitud de los estudiantes, pero esta actividad también genera dificultadas relacionadas con el estrés (Ilaja, 2016). El ejercicio docente en la actualidad continúa siendo materia de discusión, debido a que el proceso de enseñanza es uno de los factores que genera mayor estrés en los docentes universitarios (Iqbal y Abbasi, 2013). El estado actual de la inteligencia emocional todavía puede servir como referencia para mejorar aún más el rasgo de liderazgo de los docentes, con un rasgo de liderazgo mejorado, los docentes pueden enfrentar fácilmente los desafíos en sus deberes y responsabilidades. La inteligencia emocional (IE) puede ser un factor contribuyente para la mejora del rasgo de liderazgo del docente (Lucero y Ocampo, 2019). La mayoría de las investigaciones sobre docencia, tanto a nivel de educación secundaria como superior, han mostrado las características de un maestro efectivo en un conjunto de conocimiento, habilidades y actitudes proporcionalmente combinadas para el logro de los objetivos de aprendizaje (Fuentes, Sabido-Codina y Albert, 2019; Feixas, Lagos, Fernández y Sabaté, 2015). Aunque, el servicio educativo ha evolucionado a un espacio de mayor interacción como mentor-estudiante (Fernandes, Sotolongo y Martínez, 2016). Para cubrir las expectativas y necesidades de los estudiantes con conciencia social, emocional y digital, deben ser atendidos de manera madura, amigable y creativa. Por lo tanto, el docente universitario 
necesita contar con habilidades y actitudes que ayuden a relacionarse con mayor efectividad con sus estudiantes, sus pares y así mejorar su desempeño en la universidad (Bedoya, Vega, Severiche y Meza, 2017; Rodríguez-García, Sola-Martínez y Fernández-Cruz, 2017).

El docente necesita comprender y regular sus emociones, sus sentimientos y de los estudiantes para mantener un equilibrio entre el enfoque formal e informal de la enseñanza. En estudios anteriores se ha demostrado la importancia de la parte emocional en el proceso de enseñanza-aprendizaje (Penalva, Hernández y Guerrero, 2013). Asimismo, ArizaHernández, (2017) comenta la importancia de analizar las emociones en el docente debido a la vinculación con la gestión académica e investigativa. Cejudo y López-Delgado, (2017) propone que existe una relación directa y significativa entre la inteligencia emocional y el proceso de aprendizaje de los estudiantes. Palomera, Fernández-Berrocal y Brackett, (2008) reitera la importancia de estudiar las mejoras del proceso de enseñanza mediante la inteligencia emocional. Mientras Fuentes et al. (2019) han puesto de manifiesto la claridad posterior sobre las diversas emociones que enfrenta un maestro mientras enseña, En el mismo sentido Salazar, Cortez y Guillén, (2013) han declarado que la emoción es el aspecto más mencionado, pero el menos investigado de los estudios en la enseñanza. Estudios anteriores han formulado el conjunto de competencias para maestros en varios niveles en la industria de la educación. Monereo y Domínguez, (2014) identificaron las competencias de personalidad clave para los docentes universitarios, por último, Araújo y Esteves, (2016) identificaron altos nivel de compromiso docente debido a una alta percepción del bienestar psicológico, esta sensación genera mayor productividad.

La búsqueda de mejores modelos de enseñanza, ha llevado a la inclusión en los estudios de las emociones del docente tratando de entender y pronosticarlos (Estupiñan, Cherrez, Intriago y Torres, 2017). Tanto los docentes como los estudiantes deben conocer como regular sus emociones, para mejorar las relaciones interpersonales en el aula (Millán, Calvanese y D'Aubeterre, 2017). Además, los docentes reciben presiones adicionales por sus actividades administrativas y de investigación que son obligatorias, por lo tanto, se hace necesario un mejor manejo para no afectar la calidad de servicio y el bienestar de los estudiantes (Menendez, 2018). En la actualidad el estudiante está más involucrado en el mundo digital, pero más alejado de la vida social y el manejo de sus emociones. De modo que para cubrir sus necesidades los docentes deben tener una mayor preparación en el manejo y control de las emociones (Millán et al. 2017).

Las exigencias del docente en cuanto a las actividades de enseñanza, generan preocupaciones (Bisquerra y Perez, 2007). Para lograr un buen clima en el aula, el docente debe controlar las emociones negativas (De-Juan et al. 2015). Las emociones negativas pueden surgir debido a diferentes razones, las más comunes son los desacuerdos entre estudiantes y maestros que demuestran ser el principal obstáculo para el estrés y el cansancio (López-Vilchez y Gil Monte, 2015). Adicionalmente la relación con los colegas y los trámites administrativos pueden ser fuentes de conflictos (Surdez, Magaña y Sandoval, 2015). Para lograr un buen desempeño docente es necesario identificar y controlar las emociones negativas. Alanis, (2016) propone las características de un docente efectivo en la enseñanza, el control de sus pensamientos, sus actitudes y del estrés. Mayer y Salovey, (1997) sugieren como lograr el desarrollo personal mediante la percepción, comprensión y regulación de las emociones para luego convertirlo en pensamientos positivos. Cejudo y López-Delgado, (2017) han considerado que la inteligencia emocional desempeña un papel fundamental en la mejora de la eficacia de los docentes mediante el desarrollo de conocimientos, habilidades y actitudes, la autorreflexión y las habilidades de autogestión de la inteligencia emocional. Las investigaciones indican que los docentes que son más inteligentes emocionalmente tienden a manejar bien sus interacciones al preferir un tipo de 
cooperación y un estilo de evitación (Coronel y Ñaupari, 2011; Pincay-Aguilar, CandelarioSuarez y Castro-Guevara, 2018)

Existen muy pocos estudios sobre la inteligencia emocional en la educación universitaria. Sutton y Wheatley, (2003) expresan una preocupación sobre la influencia del control de las emociones del docente en el proceso de aprendizaje del estudiante. Es decir, la baja comprensión y la poca regulación de las emociones del docente generan bajo rendimiento académico (González y Berrocal, 2016). Los docentes universitarios para ser efectivos en el proceso de enseñanza y aprendizaje deben incluir a la inteligencia emocional como un factor fundamental dentro de sus capacidades profesionales (Bisquerra y Perez, 2007; Buitrón y Navarrete, 2008) El reconocimiento de la inteligencia emocional como un componente primordial para el desempeño docente, representa que el docente de ser empático con los estudiantes y promover un ambiente saludable en el aula (Mortiboys, 2012).

Las competencias con inteligencia emocional (CIE), se manifiestan mediante el proceso de escuchar y comprender las necesidades de ambas partes y proponer soluciones a situaciones de conflicto entre docentes y estudiantes, mediante las habilidades y actitudes positivas (Isaza-Zapata y Calle-Piedrahíta, 2016). También, se ha considerado que las CIE desempeña un papel crucial en la efectividad de la enseñanza de un docente (Morris, 2014; Bisquerra y Perez, 2007; Blašková, Blaško y Kucharčíková, 2014). Los CIE son esenciales no solo para mejorar la calidad de la enseñanza sino también para el desarrollo socioemocional de los estudiantes (Sutton y Wheatley, 2003). Adicionalmente las CIE promueve que los docentes busquen su autorrealización tanto personal como profesionalmente, por medio de una adecuada motivación y la satisfacción en el trabajo (Ilaja, 2016). Por último, según Cejudo y López-Delgado, (2017) la IE toca varias facetas de la actitud y el comportamiento de los docentes, lo que afecta las competencias que poseen y exhiben. Una revisión crítica de la literatura citada sugiere que la inteligencia emocional afecta las competencias de un docente para lograr niveles elevados de desempeño y mejorar la calidad de la enseñanza. La presente investigación intenta incorporar el concepto de inteligencia emocional en los docentes y cómo se puede incorporar en el desempeño de las competencias en los docentes universitarios, para lo cual se plantean los objetivos:

1. Analizar la relación de la inteligencia emocional con las competencias básicas de los docentes (conocimiento, habilidad y actitud) requeridas para la enseñanza.

2. Comprender como afecta el desempeño docente a través del desarrollo y validación de un modelo.

Las CIE para el presente estudio se derivaron de la prueba desarrollada por Chadha y Singh (2001), dos razones principales para reducir a esta prueba fueron que es relevante para el contexto peruano y, en segundo lugar, esta prueba ya ha sido probada y validada en la comunidad de docentes. La prueba se basa en la definición de IE de Menendez, (2018) "la capacidad de un individuo para responder de manera adecuada y exitosa a una gran variedad de estímulos emocionales que se generan del ser interno y del entorno inmediato"(p16). El cuestionario comprende 3 dimensiones: madurez y sensibilidad y competencia emocional las que se muestran en la (Tabla 1 ).

Por otro lado, el modelo de ecuaciones estructurales (SEM) es una herramienta estadística que combina análisis factorial y regresiones múltiples multivariadas (Hair, Hult, Ringle y Sarstedt, 2017). El modelo SEM tiene muchas aplicaciones en investigaciones en ciencias sociales, siendo aceptado por los investigadores porque considera los constructos como variables latentes observables, además puede realizarse predicciones más precisas (Sarstedt y Cheah, 2019). 
Tabla 1.

Dimensiones de la Inteligencia Emocional

\begin{tabular}{|c|c|c|}
\hline Dimensión & Características & Descripción \\
\hline \multirow{2}{*}{ Madurez } & $\begin{array}{l}\text { Conocimiento de sí } \\
\text { mismo }\end{array}$ & $\begin{array}{l}\text { Poder reconocer sentimientos de no mismo y } \\
\text { ponerles una denominación. }\end{array}$ \\
\hline & Flexibilidad & $\begin{array}{l}\text { Poder controlar emociones negativas: las } \\
\text { dudas, sospechas y celos. }\end{array}$ \\
\hline \multirow[t]{2}{*}{ Sensibilidad } & $\begin{array}{l}\text { Mejorando las relaciones } \\
\text { interpersonales }\end{array}$ & $\begin{array}{l}\text { Las relaciones interpersonales positivas } \\
\text { conducen al éxito. }\end{array}$ \\
\hline & $\begin{array}{l}\text { Transmisibilidad de las } \\
\text { emociones. }\end{array}$ & $\begin{array}{l}\text { Las emociones alegres positivas promueven la } \\
\text { confianza y el respeto propio. }\end{array}$ \\
\hline \multirow[b]{2}{*}{$\begin{array}{l}\text { Competencia } \\
\text { emocional }\end{array}$} & $\begin{array}{l}\text { Hacer frente a los } \\
\text { trastornos emocionales }\end{array}$ & $\begin{array}{l}\text { Maneja y encuentra formas de lidiar con la ira, } \\
\text { el miedo, la ansiedad y la tristeza. }\end{array}$ \\
\hline & $\begin{array}{l}\text { Responder a las } \\
\text { emociones. } \\
\text { Manejando el egoísmo }\end{array}$ & $\begin{array}{l}\text { Ser creativo y práctico hacia los impulsos } \\
\text { emocionales provocados por el ser interno. } \\
\text { Abordar los problemas del ego sin dañar a } \\
\text { nadie. Buen nivel de autoestima. }\end{array}$ \\
\hline
\end{tabular}

El objetivo principal es explicar las causas de los hechos mediante las variables latentes que, a su vez, son explicadas por las variables observables (Henseler, Ringle y Sinkovics, 2009). En la presente investigación se usó de la técnica de ecuaciones estructurales con mínimos cuadrados parciales (PLS-SEM). Por lo tanto, se plantean las hipótesis:

H1: Existe una relación causal significativa entre la inteligencia emocional y la actitud del docente.

$\mathrm{H} 2$ : Existe una relación causal significativa entre la inteligencia emocional y el conocimiento del docente.

H3: Existe una relación causal significativa entre la inteligencia emocional y las habilidades del docente.

H4: Existe una relación causal significativa entre la actitud y el desempeño docente.

H5: Existe una relación causal significativa entre el conocimiento y el desempeño docente.

H6: Existe una relación causal significativa entre las habilidades y el desempeño docente.

Se desarrolló un modelo que recopila las competencias, incluidas las competencias de la IE, actitud, habilidades y conocimientos del docente estas se relacionan con el desempeño docente. Por lo tanto, se desarrolla un modelo teórico inicial (Figura 1) que ilustra la relación entre las diversas competencias. 


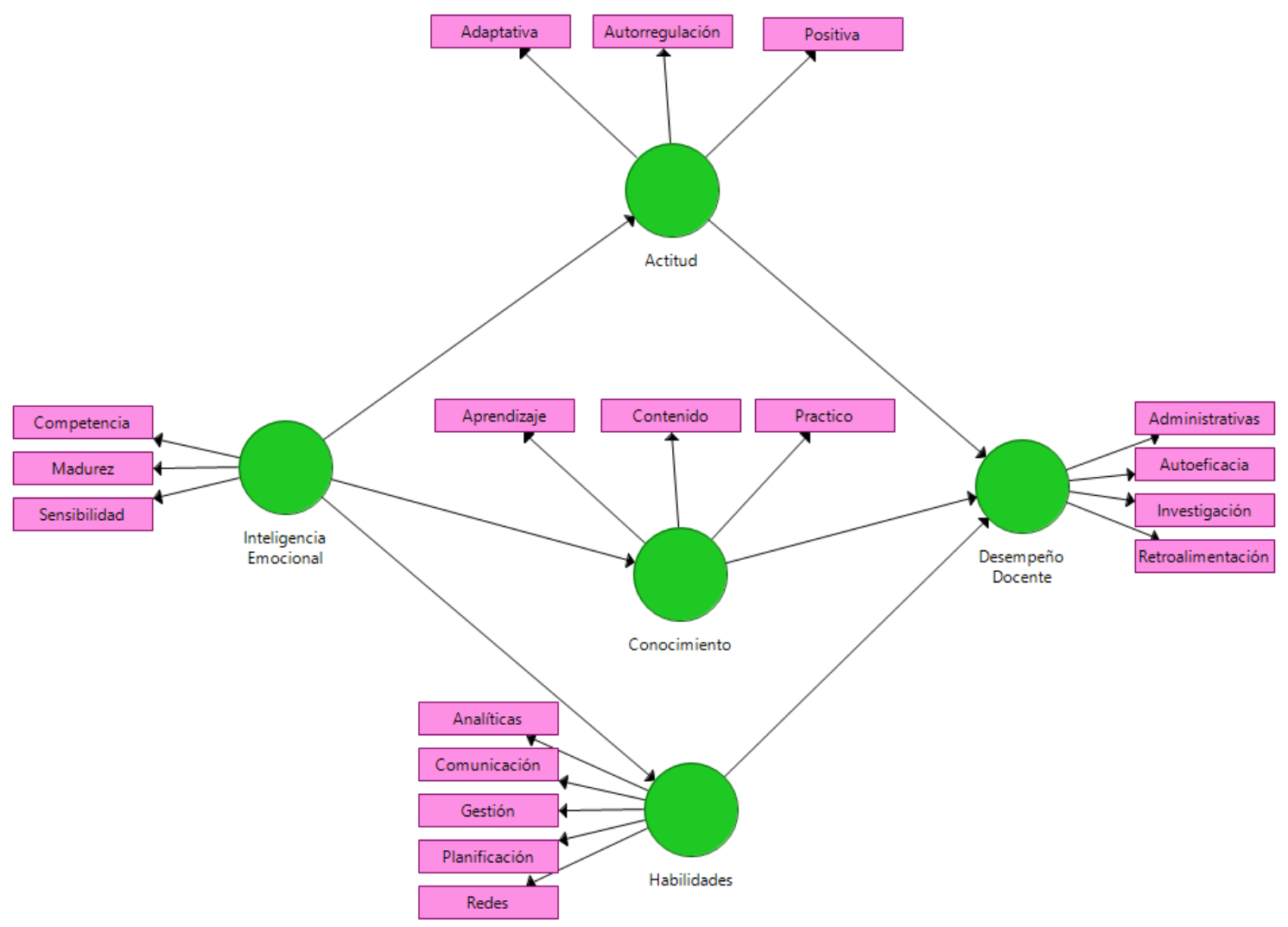

Figura 1. El modelo de propuesto inicial

\section{Metodología}

En el estudio se pretende examinar las competencias con inteligencia emocional e identificar la relación causal sobre el desempeño docente de la Universidad Nacional Santiago Antúnez de Mayolo. El tipo de estudio fue causal de enfoque cuantitativo, con diseño transversal (Hernández-Sampieri y Mendoza, 2018). Se utilizaron dos cuestionarios, uno compuesta por los datos demográficos de los encuestados como edad, sexo, años, de servicio, categoría docente y Grados académicos, y la segunda parte comprendía por cinco constructos. La variable latente inteligencia emocional la cual tenía tres variables observables estas son: madurez emocional, estabilidad emocional y competencia emocional. Contenido en la prueba de coeficiente emocional desarrollada por (Chadha y Singh, 2006). (Kaur y Shri, 2015) extrajeron las variables latentes como el conocimiento, las habilidades, la actitud y el desempeño del docente, (Mora-Cifuentes, 2016) adicionalmente, identificó las competencias básicas requeridas para una enseñanza efectiva bajo clasificaciones de conocimiento, habilidades y actitud.

En el estudio se utilizó el método de muestreo aleatorio simple. Según (Hernández-Sampieri y Mendoza, 2018) comenta que el muestreo aleatorio simple permite al investigador mantener la aleatoriedad y obtener respuestas de una manera factible, por lo tanto, Antes de realizar la encuesta, el investigador obtuvo el permiso del rector de la universidad para recopilar los datos dentro del campus universitarios. luego, los encuestadores visitaron personalmente los docentes que además firmaron un consentimiento informado para su participación y solicitaron a los docentes que llenaran el cuestionario, el tamaño de muestra requerido fue de 244 docentes que iban desde grupos de edad de 28 a más de 75 años de todas las facultades de la Universidad Nacional Santiago Antúnez de Mayolo. 
Para medir las relaciones causales entre la inteligencia emocional y las competencias del decente como son; conocimiento, habilidades, actitudes y estos a su vez influyen en el desempeño docente, los resultados han sido analizados utilizando el software SmartPLS versión 3.3.0.

\section{Resultados}

Los datos demográficos de la muestra seleccionada para lograr el propósito de este estudio fueron, los varones representan el $62.36 \%$ y mujeres $37.64 \%$. La edad de los encuestados $9.56 \%$ es para menores de 35 años, $17.34 \%$ que cuenta a la edad entre 36 a 45 años, 28.02\% para 46 a 55 años, 31.85\% para 56 a 65 años y 13.24\% encuestados de 65 a más. En cuanto a los grados académicos de los encuestados, el $16 \%$ de los encuestados tenía solo grado de bachiller, el $58 \%$ de los que tenían grado de maestría, y el $26 \%$ de cuanta con doctorado.

\section{Validación del modelo}

Para evaluar el modelo teórico del estudio, se utilizó técnica del análisis de Mínimo Cuadrado Parcial (PLS) con el uso del software SmartPLS 3.3.0 (Sarstedt y Cheah, 2019), en un esfuerzo por refinar todos los procedimientos analíticos en dos etapas del modelo de ecuaciones estructurales, donde se probó el modelo de medición y el modelo estructural recomendados por Becker, Ringle y Sarstedt, (2018), antes del estudio de modelado estructural, se realizó un análisis de las variables latentes para determinar su dimensionalidad, validez y confiabilidad utilizando el análisis factorial confirmatorio (Leyva y Trinidad, 2014).

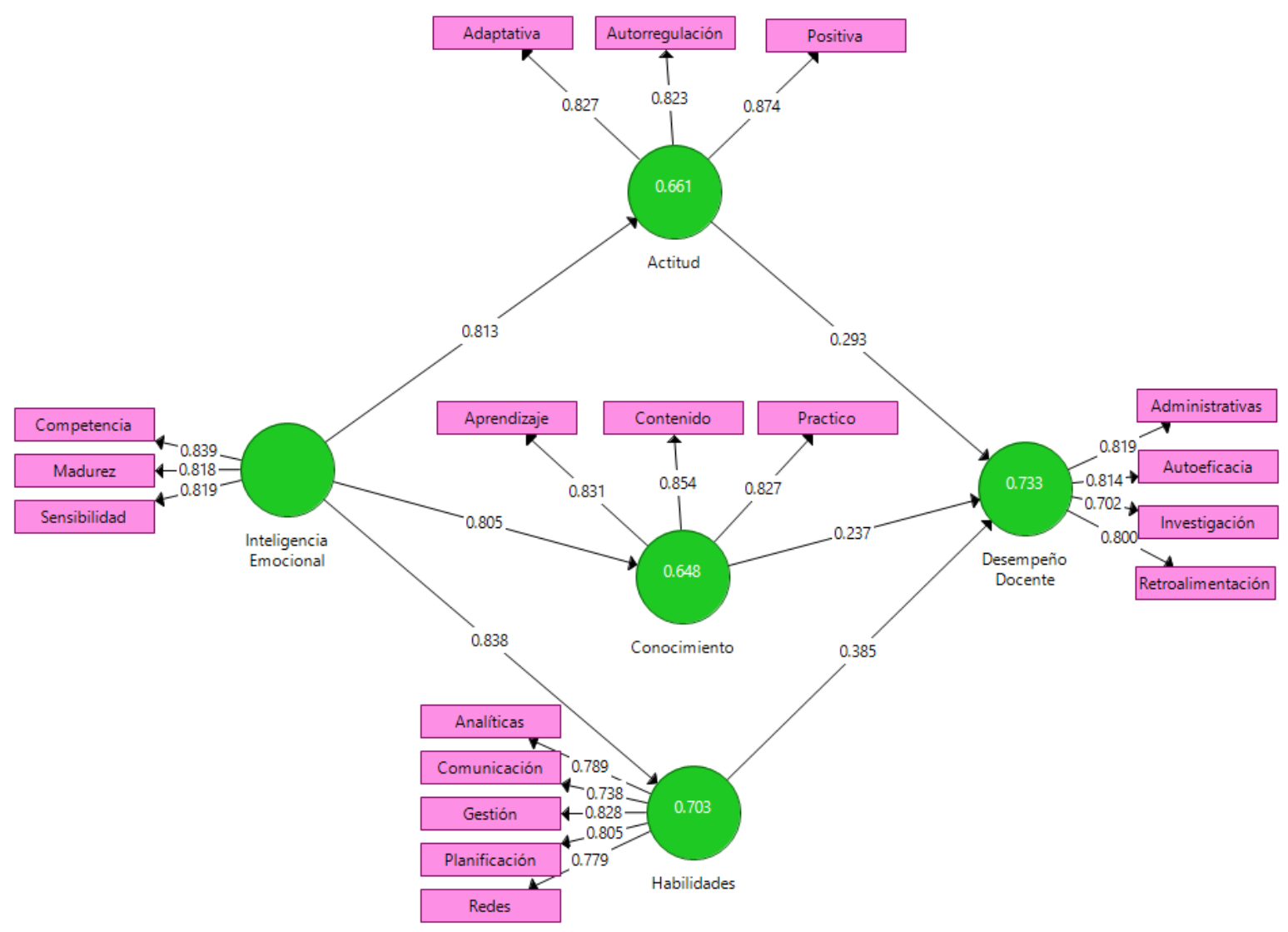

Figura 2. Relación de la inteligencia emocional, habilidades y desempeño docente. 
Por otro lado, para la comprobación de la validez de los constructos, fue necesario analizar la validez convergente del modelo general teórico se determinó analizando las cargas de los factores, la varianza promedio extraída y la confiabilidad compuesta (Ruiz, Pardo y San Martín, 2010), la figura 2 muestra los resultados de las cargas factoriales según lo recomendado por el nivel umbral de 0.6, según Henseler et al. (2009). Todos los valores estuvieron por encima de 0.6 , lo que muestra la validez convergente del modelo.

\section{Validez convergente}

En cuanto a la validez convergente se mide a través de la estimación de la varianza promedio extraído (AVE), lo cual debe ser un valor mayor que 0.5 (Becker et al. 2018). La varianza media extraída representa la totalidad de la varianza de las variables observables acumulada en las variables latentes. Cuando el valor del AVE sea mayor, mejor representación tendrá la carga de las variables observables. En la tabla 2 también se presenta el alfa de Cronbach $(\alpha)$, las cargas externas y los grados de fiabilidad compuesta (FC) donde el indicador (variables observables) del constructo representa a la variable latente, los valores superaron el 0.7 recomendado por Fornell y Larcker, (1981).

Tabla 2.

Resultados del modelo de medición

\begin{tabular}{|c|c|c|c|c|}
\hline Constructo & $\begin{array}{l}\text { Cargas } \\
\text { externas }\end{array}$ & $\begin{array}{c}\text { Alfa de } \\
\text { Cronbach }(\alpha)\end{array}$ & $\begin{array}{c}\text { Fiabilidad } \\
\text { compuesta (FC) }\end{array}$ & $\begin{array}{c}\text { Varianza extraída } \\
\text { media (AVE) }\end{array}$ \\
\hline Actitud & Actitud & 0.794 & 0.879 & 0.709 \\
\hline Adaptativa & 0.827 & & & \\
\hline Autorregulación & 0.823 & & & \\
\hline Positiva & 0.874 & & & \\
\hline Conocimiento & Conocimiento & 0.787 & 0.876 & 0.702 \\
\hline Aprendizaje & 0.831 & & & \\
\hline Contenido & 0.854 & & & \\
\hline Practico & 0.827 & & & \\
\hline Desempeño Docente & Desempeño & 0.792 & 0.865 & 0.617 \\
\hline Administrativas & 0.819 & & & \\
\hline Autoeficacia & 0.814 & & & \\
\hline Investigación & 0.702 & & & \\
\hline Retroalimentación & 0.800 & & & \\
\hline Habilidades & Habilidades & 0.848 & 0.891 & 0.622 \\
\hline Analíticas & 0.789 & & & \\
\hline Comunicación & 0.738 & & & \\
\hline Gestión & 0.828 & & & \\
\hline Planificación & 0.805 & & & \\
\hline Redes & 0.779 & & & \\
\hline Inteligencia Emocional & $\mathrm{IE}$ & 0.766 & 0.865 & 0.681 \\
\hline Competencia & 0.839 & & & \\
\hline Madurez & 0.818 & & & \\
\hline Sensibilidad & 0.819 & & & \\
\hline
\end{tabular}




\section{Validez discriminante}

La validez discriminante, nivel en la cual los elementos se diferencian entre constructos, es decir, muestra que tanto una variable (constructo) se diferencias de las otras variables (Martínez y Fierro, 2018), La raíz cuadrada de la varianza media extraída se resalta en negrita y están en las diagonales, este valor fue mayor que los valores de cada fila y cada columna respectivamente.

Tabla 3

Validez discriminante del modelo de medición

\begin{tabular}{lccccc}
\hline \multicolumn{1}{c}{ Constructo } & Actitud & Conocimiento & Desempeño & Habilidades & $\begin{array}{c}\text { Inteligencia } \\
\text { Emocional }\end{array}$ \\
\hline Actitud & 0.842 & & & & \\
Conocimiento & 0.777 & 0.838 & & & \\
Desempeño & 0.778 & 0.785 & 0.821 & & \\
Habilidades & 0.807 & 0.832 & 0.819 & 0.839 & \\
Inteligencia Emocional & 0.813 & 0.805 & 0.802 & 0.818 & 0.826 \\
\hline
\end{tabular}

\section{Cargas cruzadas}

La validez discriminante se mide a través del análisis de las cargas cruzadas de los indicadores (Leyva y Trinidad, 2014), se realizó comparando las cargas externas de un indicador en las construcciones asociadas y debe ser mayor que toda su carga en las otras construcciones (Ruiz et al. 2010), los valores se muestran en la tabla 4.

Tabla 4.

Cargas cruzadas de variables latentes y observables

\begin{tabular}{lccccc}
\hline \multicolumn{1}{c}{ Ítems } & Actitud & Conocimiento & Desempeño & Habilidades & $\begin{array}{c}\text { Inteligencia } \\
\text { Emocional }\end{array}$ \\
\hline Adaptativa & $\mathbf{0 . 8 2 7}$ & 0.682 & 0.706 & 0.704 & 0.697 \\
Autorregulación & $\mathbf{0 . 8 2 3}$ & 0.584 & 0.623 & 0.648 & 0.661 \\
Positiva & $\mathbf{0 . 8 7 4}$ & 0.692 & 0.658 & 0.682 & 0.693 \\
Aprendizaje & 0.636 & $\mathbf{0 . 8 3 1}$ & 0.655 & 0.680 & 0.655 \\
Contenido & 0.683 & $\mathbf{0 . 8 5 4}$ & 0.684 & 0.703 & 0.697 \\
Practico & 0.633 & $\mathbf{0 . 8 2 7}$ & 0.633 & 0.708 & 0.669 \\
Administrativas & 0.631 & 0.648 & $\mathbf{0 . 8 1 9}$ & 0.672 & 0.681 \\
Autoeficacia & 0.640 & 0.630 & $\mathbf{0 . 8 1 4}$ & 0.679 & 0.637 \\
Investigación & 0.530 & 0.539 & $\mathbf{0 . 7 0 2}$ & 0.536 & 0.562 \\
Retroalimentación & 0.665 & 0.642 & $\mathbf{0 . 8 0 0}$ & 0.673 & 0.635 \\
Analíticas & 0.620 & 0.663 & 0.671 & $\mathbf{0 . 7 8 9}$ & 0.658 \\
Comunicación & 0.613 & 0.645 & 0.584 & $\mathbf{0 . 7 3 8}$ & 0.595 \\
Gestión & 0.686 & 0.697 & 0.681 & $\mathbf{0 . 8 2 8}$ & 0.709 \\
Planificación & 0.636 & 0.645 & 0.650 & $\mathbf{0 . 8 0 5}$ & 0.677 \\
Redes & 0.626 & 0.631 & 0.638 & $\mathbf{0 . 7 7 9}$ & 0.662 \\
Competencia & 0.683 & 0.672 & 0.670 & 0.702 & $\mathbf{0 . 8 3 9}$ \\
Madurez & 0.658 & 0.689 & 0.684 & 0.704 & $\mathbf{0 . 8 1 8}$
\end{tabular}


0.632

0.632

0.670

0.819

\section{Contrastación de Hipótesis}

Posterior a conseguir el modelo de medición, las hipótesis se probaron realizando el procedimiento de arranque con una muestra de 500, como lo sugirieron Hair et al. (2017), la tabla 5 muestra los resultados de la contrastación de hipótesis, se puede ver que las seis hipótesis tienen relacion causal significativa con sus respectivas variables latentes. Se obtuvieron las estadísticas t correspondientes, las estadísticas T de valores superiores a 1,96 (con una confianza del 95\%) indican la importancia de los parámetros, en el modelo, todos los casos mostraron un valor superior a 1,96. A continuacion, la inteligencia emocional tiene relación causal significativa en la actitud del docente, $\mathrm{H} 1$ : $(\beta=0.813, \mathrm{t}=24.111, \mathrm{p}<0.001)$; del mismo modo, la inteligencia emocional tiene relación causal significativa en el conocimiento, $\mathrm{H} 2:(\beta=0.805, t=22.699, p<0.001)$; La hipotesos $\mathrm{H} 3$ la inteligencia emocional tiene relación causal significativa en las habilidades del docente está respaldada por $(\beta=0.838, t=27.681$, $\mathrm{p}<0.001)$; de la misma manera, la actitud tiene relación causal significativa en el desempeño docente $\mathrm{H}_{4}$ : $(\beta=0.293, t=4.453, p<0.001)$; La hipotesos $\mathrm{H}_{5}$ el conocimiento tiene relación causal significativa en el desempeño docente está respaldada por $(\beta=0.237, t=3.377, p$ <0.01); para finalizar, la hipotesis $\mathrm{H} 6$ las habilidades tiene relación causal significativa en el desempeño docente devido de los valores $(\beta=0.385, t=5.143, p<0.001)$. Esto demuestra la validez del modelo de comptencias de inteligencia eomcional en el docente unviersitario. Además, se utilizó el $R^{2}$ para medir el tamaño del efecto. El $R^{2}$ para la actitud del docente de 0.661 , El $R^{2}$ para el conocimiento del docente de 0.648 , El $R^{2}$ para las habilidades del docente de 0.703 y El $R 2$ para el desempeño docente de 0.733 . estos valores son considerados sustanciales según Cohen, (1988) sugirió que los valores de R cuadrado de 0.67, 0.33 y 0.19 se consideran sustanciales, moderados y débiles, respectivamente. Por último, el residuo estandarizado cuadrático medio (SRMR) fue 0,056 y se considera acaptable. Según Chin, (1998) un valor de SRMR < 0,08 es aceptable y SRMR < 0,05 es óptimo.

Tabla 5.

Coeficientes de rutas del modelo

\begin{tabular}{llccrr}
\hline $\mathbf{N}^{\circ}$ & \multicolumn{1}{c}{ Descripción } & $\begin{array}{c}\text { Coef. } \\
\text { de ruta } \\
(\boldsymbol{\beta})\end{array}$ & $\begin{array}{c}\text { Desv. } \\
\text { estándar } \\
(\boldsymbol{\sigma})\end{array}$ & $\begin{array}{c}\text { Estadístico } \\
\mathbf{t}\end{array}$ & P valor \\
\hline $\mathrm{H} 1$ & Inteligencia Emocional => Actitud & 0.813 & 0.034 & 24.111 & $0.000^{* * *}$ \\
$\mathrm{H} 2$ & Inteligencia Emocional => Conocimiento & 0.805 & 0.035 & 22.699 & $0.000^{* * *}$ \\
$\mathrm{H} 3$ & Inteligencia Emocional => Habilidades & 0.838 & 0.030 & 27.681 & $0.000^{* * *}$ \\
H4 & Actitud => Desempeño docente & 0.293 & 0.066 & 4.453 & $0.000^{* * *}$ \\
H5 & Conocimiento => Desempeño docente & 0.237 & 0.070 & 3.377 & $0.001 * *$ \\
H6 & Habilidades => Desempeño docente & 0.385 & 0.075 & 5.143 & $0.000^{* * *}$ \\
\hline
\end{tabular}

Nota: Nivel de significancia donde, ${ }^{*} \mathrm{p}<0.05,{ }^{* *} \mathrm{p}<0.01, * * * \mathrm{p}<0.001$.

\section{Discusión}

Comprender el papel de la IE en la enseñanza: los resultados se analizaron para estimar la IE de los docentes bajo tres dimensiones: sensibilidad, madurez y competencia emocional. Las estadísticas descriptivas simples indican que la IE de los docentes fue considerado de nivel medio según la prueba de EQ administrada. La puntuación media de sensibilidad fue (medio), 
la puntuación media de madurez emocional fue (medio) y la puntuación media de competencia emocional fue (alta). Los resultados sugieren que la mayoría de los encuestados exhiben más competencias emocionales como autorregulación, manejo del egoísmo y lucha contra los trastornos emocionales. En cambio, la madurez emocional del docente fue medio, esto apoya la posición de Salazar et al. (2013) que menciona que la enseñanza debe tener como centro el crecimiento del estudiante y adaptarse a diversas reacciones en el aula. También Ansari y Malik, (2017) expone que el docente debe tener empatia para mantener una comunicación fluida con los estudiantes. Es decir que el docente debe reconocer las emociones de los estudiantes y tratar de controlar las reacciones para obtener la confianza de los estudiantes (Alanis, 2016). También se observó que la madurez más alta se mostraba en el grupo de edad de 65 años o más, Esto puede atribuirse al hecho de que a medida que el profesor gana experiencia, crece con la edad y se hace más maduro, está en una mejor posición para analizarse a sí mismo (Ovans, 2015). Quiere decir que los docentes han logrado sus objetivos a nivel profesional y tienen una estabilidad personal (Cejudo y López-Delgado, 2017). En este perido de vida, los docentes tiene una actitud con mayor equilibrio, flexibilidad y adaptabilidad. Tambien obtuvieron niveles altos de sensiblidad, es decir los docentes de mayor edad comprenden mejor a sus estudiantes (Ariza-Hernández, 2017).

El grupo de edad menores a 35 años han ontenido puntajes muy bajos en la competencia emocional, posiblemente es a causa de la presion por lograr un nombramiento y tener cierta estabilidad en el trabajo. Este resultado se mililar a mostrado por Coronel y Ñaupari, (2011) y comenta que los profesionales en la actualidad tienen conflictos entre sus intereses persoales y profesionales, además los docentes auxiliares contratados muestran un nivel bajo de control de la inteligencia emocional, debido a que se encuentran en el inicio de su carrera docente y ni pueden controlo su ego. Si observamos los datos con perspectiva de género, se observó que las mujeres ocupaban el puesto más alto en sensibilidad y carecían de competencia. Los resultados coinciden con los estudios previos y las nociones de que las mujeres son emocionalmente más sensibles y menos prácticas (Millán et al. 2017; Menendez, 2018).

Relación entre El y las competencias docentes: los datos se han analizado mediante el modelado de rutas mediante el enfoque de mínimos cuadrados parciales (PLS) de SEM utilizando el software SmartPLS. la validez convergente se midió a través de la estimación de la varianza promedio extraído (AVE), lo cual debe ser un valor mayor que 0.5 según Becker et al. (2018). Cuando el valor del AVE sea mayor, mejor representación tendrá la carga de las variables observables. también se utilizó el alfa de Cronbach $(\alpha)$, las cargas externas y los grados de fiabilidad compuesta (FC) donde el indicador (variables observables) del constructo representa a la variable latente, los valores superaron el 0.7 recomendado por Fornell y Larcker, (1981).

Los resultados en el modelo SEM (Figura 2) muestran que la IE tiene un efecto muy alto en las competencias de habilidad de un maestro (0.838); en las competencias de actitud de un maestro (0.813) y las competencias de conocimiento de un maestro (0.805). Las competencias relacionadas con las habilidades tienen los mayores contribuyentes al desempeño docente (0.385). La actitud tiene un efecto razonable en el rendimiento con un valor de coeficiente de trayectoria de 0.293 . Sin embargo, el conocimiento contribuye menos a un rendimiento docente si se considera solo $(0,237)$. Quier decir que no es sufiente tener muchos conocimientos, es necesario combinarlo con las habilidades y demostrar las actitudes positivas ante los estudiantes, este resultado concuerda con Pincay-Aguilar et al. (2018) expone para llegar a la efectividad del docente se necesita mas que transmitir conocimientos, es importente el como se logra la atencion del estudiante. Según Bisquerra y Perez, (2007), un maestro con IE mejorado podrá difundir mejor su conocimiento, 
comprender las necesidades de los estudiantes, se preocupará más por ellos y ganará su confianza, también Mora-Cifuentes, (2016) menciona que la IE puede ayudar a alcanzar nuevos niveles en su propia autorrealización a través de lograr mejores redes y relaciones. Por lo tanto, la IE puede resultar beneficiosa no solo para su relación con los estudiantes sino también para su propio crecimiento (Millán et al. 2017).

\section{Conclusiones}

Las competencias básicas de enseñanza son; conocimiento, comunicaicon y autonomia estos son importantes para ser un docente efectivo, pero no completo. Debido a que pueden exsitir otras variables que esten relacionadas con el desempeño docente, Este vacío se llena con las competencias con inteligencia emocional que impactan las habilidades, la actitud y el conocimiento de un docente para producir un desempeño eficiente, este modelo establece la importancia de la inteligencia emocional en el proceso de enseñanza, mediante la mejora de sus competencias: Conocimientos, habilidades y actitudes en el logro de un mejor desempeño docente.

La inteligencia emocional debe ser reconocida como un componente esencial del conjunto de competencias de los docentes y no una mera cualidad adicional y debe recibir una atención igual a la del contenido y la pedagogía.

\section{Referencias}

Alanis, J. F. (2016). Excelencia y profesores universitarios. En D. Izarra, Experiencias y perspectivas en ética profesional y responsabilidad social universitaria en Iberoamérica (págs. 126-141). Caracas: Centro de Investigación Educativa Georgina Calderón. Recuperado de https://dialnet.unirioja.es/servlet/articulo?codigo=5750605

Ansari, A. H., \& Malik, S. (2017). Ability-based emotional intelligence and knowledge sharing. VINE Journal of Information and Knowledge Management Systems, 47(2), 211-227. https://doi.org/10.1108/VJIKMS-09-2016-0050

Araújo, I., \& Esteves, R. (2016). Engagement em docentes do ensino superior: uma abordagem exploratória. Enfermería Universitaria, 13(2), 73-79. https://doi.org/10.1016/j.reu.2016.03.006

Ariza-Hernández, M. L. (2017). Influencia de la inteligencia emocional y los afectos en la relación maestro-alumno, en el rendimiento académico de estudiantes de educación superior. Educación y educadores, 20(2), 193-210. https://doi.org/10.5294/edu.2017.20.2.2

Becker, J.-M., Ringle, C., \& Sarstedt, M. (2018). Estimating moderating effects in PLS-SEM and PLSc-SEM: Interaction term generation data treatment. Journal of Applied Structural Equation Modeling, 2(2), 1-21. Recuperado de https://jasemjournal.com/wpcontent/uploads/2019/10/Becker-et-al-2018-JASEM.pdf

Bedoya, E. A., Vega, N. E., Severiche, C. A., \& Meza, M. J. (2017). Síndrome de Quemado (Burnout) en docentes universitarios: El caso de un centro de estudios del Caribe Colombiano. Formación universitaria, 10(6), 51-58. http://dx.doi.org/10.4067/S071850062017000600006

Bisquerra, R., \& Perez, N. (2007). Las competencias emocionales. Educación XX1, 10(1), 61-82. https://doi.org/10.5944/educxx1.10.1 
Blašková, M., Blaško, R., \& Kucharčíková, A. (2014). Competences and competence model of university teachers. Procedia-Social and Behavioral Sciences, 159(23), 457-467. https://doi.org/10.1016/j.sbspro.2014.12.407

Buitrón, S., \& Navarrete, P. (2008). El docente en el desarrollo de la inteligencia emocional: reflexiones y estrategias. Revista Digital de Investigación en Docencia Universitaria, 4(1), 1-8. https://doi.org/10.19083/ridu.4.8

Cejudo, J., \& López-Delgado, M. L. (2017). Importancia de la inteligencia emocional en la práctica docente: un estudio con maestros. Psicología educativa, 23(1), 29-36. https://doi.org/10.1016/j.pse.2016.11.001

Chadha, N. K., \& Singh, D. (2006). Know your EQ: Emotional Quotient Test. En D. Singh, Emotional intelligence at work: a professional guide (3ra ed., págs. 210-221). New Delhi: Response Books.

Chin, W. W. (1998). Commentary: Issues and opinion on structural equation modeling. MIS Quarterly - JSTOR, 22(1), vii-xvi. Recuperado de https://www.jstor.org/stable/249674

Cohen, J. (1988). Statistical power analysis for the behavioral sciences. Nueva Jersey, USA: Lawrence Earlbaum Associates, Publishers. Recuperado de http://www.utstat.toronto.edu/ brunner/oldclass/378f16/readings/CohenPower.pdf

Coronel, E., \& Ñaupari, F. (2011). Relación entre inteligencia emocional y desempeño docente en aula en la Universidad Continental de Huancayo. Apuntes de Ciencia \& Sociedad, 1(1), 27-32. https://doi.org/10.18259/acs.2011006

De-Juan, M. D., González, E., López, J. J., Poveda, V. R., Cachero, C., Vallés, M. L., . . Peris, J. E. (2015). Una visión de 360 grados sobre la actitud hacia la diversidad en el entorno universitario. En M. T. Tortosa, J. D. Alvarez, \& B. N. Pellín, XIII Jornadas de Redes de Investigación en Docencia Universitaria (págs. 3066-3082). Alicante: Universidad de Alicante.

Estupiñan, R., Cherrez, I. M., Intriago, G. C., \& Torres, R. J. (2017). Neurociencia cognitiva e inteligencia emocional. La gestión pedagógica en el contexto de la formación profesional.Revista Didasc@ lia: Didáctica y Educación, 7(4), 207-214. Recuperado de http://refcale.uleam.edu.ec/index.php/didascalia/article/view/1511

Feixas, M., Lagos, P., Fernández, I., \& Sabaté, S. (2015). Modelos y tendencias en la investigación sobre efectividad, impacto y transferencia de la formación docente en educación superior. Educar, 51(1), 81-107. Recuperado de http://www.redalyc.org/articulo.oa?id=342133060005

Fernandes, D. J., Sotolongo, M., \& Martínez, C. C. (2016). La evaluación del desempeño por competencias: percepciones de docentes y estudiantes en la educación superior. Formación universitaria, 9(5), 15-24. http://dx.doi.org/10.4067/S071850062016000500003

Fornell, C., \& Larcker, D. F. (1981). Evaluating structural equation models with unobservable variables and measurement error. Journal of marketing research, 18(1), 39-50. https://doi.org/10.2307/3151312

Fuentes, C., Sabido-Codina, J., \& Albert, J. M. (2019). El Desarrollo de la competencia social y ciudadana y la utilización de metodologías didácticas activas en las aulas de secundaria. Revista Electrónica Interuniversitaria de Formación del Profesorado, 22(2), 199-210. https://doi.org/10.6018/reifop.22.2.369671 
González, R. C., \& Berrocal, P. F. (2016). Programas para enseñar la inteligencia emocional en las escuelas. Ideas para una adecuada implementación. Padres y Maestros / Journal of Parents and Teachers, (368), 11-17. https://doi.org/10.14422/pym.i368.y2016.002

Hair, J., Hult, T., Ringle, C., \& Sarstedt, M. (2017). A Primer on Partial Least Square Structural Equation Modeling (PLS-SEM) (2da ed.). California, USA: Sage Publications, inc.

Henseler, J., Ringle, C., \& Sinkovics, R. (2009). The use of partial least squares path modeling in international marketing. En R. Sinkovics, \& P. Ghauri, New Challenges to International Marketing (Advances in International Marketing, Vol. 20) (págs. 277319). Bingley, Reino Unido: Emerald Group Publishing Limited. https://doi.org/10.1108/S1474-7979(2009)0000020014

Hernández-Sampieri, R., \& Mendoza, C. (2018). Metodología de la investigación. Las rutas cuantitativa, cualitativa y mixta. Mexico: Mc Graw Hill.

Ilaja, B. R. (2016). Burnout y estrategias de inteligencia emocional en profesores universitarios: implicaciones en la salud laboral educativa. Psicología desde el Caribe, 33(1), 31-46. Recuperado de https://www.redalyc.org/pdf/213/21345152003

Iqbal, F., \& Abbasi, F. (2013). Relationship between emotional intelligence and job burnout among universities professors. Asian journal of social sciences \& humanities, 2(2), 219229. Recuperado de http://www.ajssh.leenaluna.co.jp/AJSSHPDFs/Vol.2(2)/AJSSH2013(2.2-24).pdf

Isaza-Zapata, G. M., \& Calle-Piedrahíta, J. S. (2016). Un acercamiento a la comprensión del perfil de la Inteligencia Emocional. Revista Latinoamericana de Ciencias Sociales, Niñez y Juventud, 14(1), 331-345. https://doi.org/10.11600/1692715x.14122220814

Kaur, I., \& Shri, C. (2015). Effective teaching competencies: A compilation of changing expectations from students and institutions. Journal of Contemporary Research in Management, $10(1), \quad 57-71 . \quad$ Recuperado de https://www.psgjcrm.com/journals/index.php/jcrm/article/view/413/286

Leyva, O., \& Trinidad, J. (2014). Modelo de ecuaciones estructurales por el método de mínimos cuadrados parciales (Partial Least Squares-PLS). En K. Sáenz, \& G. Tamez, Métodos y técnicas cualitativas y cuantitativas aplicables a la investigación en ciencias sociales (págs. 480-497). México, D.F: Tirant Humanidades. Recuperado de http://eprints.uanl.mx/id/eprint/8583

López-Vilchez, J., \& Gil Monte, P. R. (2015). Sobrecarga laboral y de gestión del personal docente en el entorno universitario actual en España. Arxius de Ciències Socials, (32), 111-120. Recuperado de https://www.uv.es/unipsico/pdf/Publicaciones/Articulos/03_RRPS/2015_Arxius.pdf

Lucero, L. C., \& Ocampo, J. M. (2019). Emotional Intelligence and Leadership Trait among Master Teachers. Mimbar pendidikan, 4(1), 55-72. Recuperado de http://www.journals.mindamas.com/index.php/mimbardik/article/viewFile/1119/998

Martínez, M., \& Fierro, E. (2018). Aplicación de la técnica PLS-SEM en la gestión del conocimiento: un enfoque técnico práctico. RIDE. Revista Iberoamericana para la Investigación y el Desarrollo Educativo, 8(16), 130-164. https://doi.org/10.23913/ride.v8i16.336

Mayer, J. D., \& Salovey, P. (1997). What is emotional intelligence? En P. Salovey, \& D. J. Sluyter, Emotional development and emotional intelligence: Educational implications (págs. 3-31). New York: Harper Collins. 
Menendez, D. (2018). Aproximación crítica a la Inteligencia Emocional como discurso dominante en el ámbito educativo. revista española de pedagogía, 76(269), 7-23. https://doi.org/10.22550/rep76-1-2018-01

Millán, A., Calvanese, N., \& D'Aubeterre, M. E. (2017). Condiciones de trabajo, estrés laboral, dependencia universitaria y bienestar psicológico en docentes universitarios. REDU. Revista de Docencia Universitaria, 15(1), 195-218. https://doi.org/10.4995/redu.2017.6009

Monereo, C., \& Domínguez, C. (2014). La identidad docente de los profesores universitarios competentes. $\quad$ Educación $\quad X X 1, \quad 17(2), \quad$ 83-104. https://doi.org/10.5944/educxx1.17.2.11480

Mora-Cifuentes, P. M. (2016). Conocimientos, habilidades y actitudes de los docentes constructores de paz. Mundo FESC, 6(11), 72-79. Recuperado de https://www.fesc.edu.co/Revistas/OJS/index.php/mundofesc/article/view/84

Morris, M. V. (2014). La Neuroeducacion en el aula: Neuronas espejo y la empatia docente. La Vida \& La Historia, (3), 7-18. https://doi.org/10.33326/26176041.2014.3.364

Mortiboys, A. (2012). Teaching with Emotional Intelligence. London: Routledge, https://doi.org/10.4324/9780203806463

Ovans, A. (2015). How emotional intelligence became a key leadership skill. Harvard Business Review 28, 3-6. Recuperado de http://workingconversations.com/wpcontent/uploads/2018/02/HowEmotionallntelligenceBecameAKeyLeadershipSkill.pdf

Palomera, R., Fernández-Berrocal, P., \& Brackett, M. A. (2008). La inteligencia emocional como una competencia básica en la formación inicial de los docentes: algunas evidencias. Electronic journal of research in educational psychology, 6(2), 437-454. Recuperado de http://www.redalyc.org/articulo.oa?id=293121924010

Penalva, A., Hernandez, M. Á., \& Guerrero, C. (2013). La gestión eficaz del docente en el aula. Un estudio de caso. Revista electrónica interuniversitaria de formación del profesorado, 16(2), 77-92. https://doi.org/10.6018/reifop.16.2.180931

Pincay-Aguilar, I., Candelario-Suarez, G., \& Castro-Guevara, J. (2018). Inteligencia Emocional en el desempeño docente. Psicología UNEMI, 2(2), 32-40. https://doi.org/10.29076/issn.2602-8379vol2iss2.2018pp32-40p

Rodríguez-García, A., Sola-Martínez, T., \& Fernández-Cruz, M. (2017). Impacto del Burnout en el desarrollo profesional del profesorado universitario. Una revisión de la investigación. Revista Electrónica Interuniversitaria de Formación del Profesorado, 20(3), 161-178. https://doi.org/10.6018/reifop.20.3.275121

Ruiz, M. A., Pardo, A., \& San Martín, R. (2010). Modelos de ecuaciones estructurales. Papeles del psicólogo, 31(1), 34-45. Recuperado de http://www.redalyc.org/articulo.oa?id=77812441004

Salazar, R. E., Cortez, G. E., \& Guillén, M. (2013). La identidad docente, la política y gobernabilidad en educación superior. European Scientific Journal, 9(2), 88-99. Recuperado de http://citeseerx.ist.psu.edu/viewdoc/download?doi=10.1.1.824.3065\&rep=rep1\&type $=p d f$ 
Sarstedt, M., \& Cheah, J.-H. (2019). Partial least squares structural equation modeling using SmartPLS: a software review. Journal of Marketing Analytics, 7(3), 162-202. https://doi.org/10.1057/s41270-019-00058-3

Surdez, E. G., M. D., \& Sandoval, M. D. (2015). Conflicto de rol en profesores universitarios integrantes de cuerpos académicos. Perfiles educativos, 37(147), $103-125$. https://doi.org/10.1016/j.pe.2013.09.001

Sutton, R. E., \& Wheatley, K. F. (2003). Teachers' emotions and teaching: A review of the literature and directions for future research. Educational psychology review, 15(4), 327-358. https://doi.org/10.1023/A:1026131715856

Uitto, M., Jokikokko, K., \& Estola, E. (2015). Virtual special issue on teachers and emotions in Teaching and teacher education (TATE) in 1985-2014. Teaching and Teacher Education, 50, 124-135. https://doi.org/10.1016/j.tate.2015.05.008 